\title{
Simulation based comparison of routing protocols in wireless multihop ad hoc networks
}

\author{
Ali H. Wheeb ${ }^{1}$, Marwa T. Naser ${ }^{2}$ \\ ${ }^{1}$ College of Enginerring, University of Baghdad, Iraq \\ ${ }^{2}$ Department of Computer Engineering, University of Baghdad, Iraq
}

\section{Article Info \\ Article history: \\ Received Sep 4, 2020 \\ Revised Oct 13, 2020 \\ Accepted Dec 5, 2020}

\section{Keywords:}

Ad hoc networks

AODV

AOMDV

DSDV

Routing protocols

\begin{abstract}
Routing protocols are responsible for providing reliable communication between the source and destination nodes. The performance of these protocols in the ad hoc network family is influenced by several factors such as mobility model, traffic load, transmission range, and the number of mobile nodes which represents a great issue. Several simulation studies have explored routing protocol with performance parameters, but few relate to various protocols concerning routing and quality of service (QoS) metrics. This paper presents a simulation-based comparison of proactive, reactive, and multipath routing protocols in mobile adhoc networks (MANETs). Specifically, the performance of AODV, DSDV, and AOMDV protocols are evaluated and analyzed in the presence of varying the number of mobile nodes, pause time, and traffic connection numbers. Moreover, Routing and QoS performance metrics such as normalized routing load, routing packet, packet delivery ratio, packet drop, end-to-end delay, and throughput are measured to conduct a performance comparison between three routing protocols. Simulation results indicate that AODV outperforms the DSDV and AOMDV protocols in most of the metrics. AOMDV is better than DSDV in terms of end-to-end delay. DSDV provides lower throughput performance results. Network topology parameters have a slight impact on AODV performance.
\end{abstract}

This is an open access article under the CC BY-SA license.

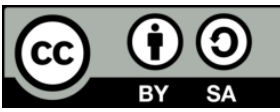

Corresponding Author:

Ali H. Wheeb

College of Engineering

University of Baghdad, Iraq

Email: ali.h.wheeb@gmail.com

\section{INTRODUCTION}

Due to the rapid development of spectacular new hardware such as intelligent drones UAVs, Internet-of-vehicles, smart mobiles, and embedded plat-forms software, the field of ad hoc networks attracted great attention from the researcher community in recent years [1]. Mobile ad hoc networks (MANETS) is a non-centralized network consists of a group of mobile nodes equipped with the wireless hardware interface that allows it to communicate via a wireless link, and have capabilities of generating own packets and forwarding receiving packets. These infrastructures-less networks expand the wireless range by the support of the multi-hop organization on the fly without any central management. [2]. MANETs can be deployed and easily and quickly to overcome the problems, making them suitable for different applications such as military applications [3], disaster area networks [4], and rescue operations [5]. Further, MANETs have been used to connect modern systems like the internet of things (IoT) [6].

The main function of routing protocols is to discover the correct and efficient route from a source to a destination node. Additionally, quality of service (QoS) routing is an essential function in MANETs to ensure end-to-end quality [7]. Therefore, MANETs need efficient and reliable routing protocols to properly 
depict the multi-hop route packets, network dynamic topology and adapt to the various changes [8]. Several routing protocols have been designed and developed for ad hoc networks. Usually, they divide into three categories proactive, reactive, and hybrid routing protocols. Ad hoc on-demand distance vector (AODV) is an on-demand reactive single path routing protocol whereas destination-sequent distance vector (DSDV) is a popular existing table-driven proactive routing protocols, whilst Ad hoc on-demand multipath distance vector (AOMDV) is an example of a reactive on-demand routing protocol.

In recent decades, several research papers focused on the study of the performance analysis and comparison of routing protocols in ad hoc networks. Most of these studies include different types of routing protocols, with varying network topology and mobility. The performance of AODV, DSDV, and Ant HocNet routing protocols investigated in the mobile ad hoc network. Simulation study implemented using network simulator NS-2 with varying scenarios. Results show that the packet delivery ratio of AODV and Ant HocNet is significantly better than the DSDV protocol [9]. Proactive and Reactive routing protocols were evaluated on MANETs. Two simulation scenarios implemented by varying network density and mobility speed. simulation results indicate that in large-scale network AODV offers better performance than DSR, while OLSR outperforms other protocols in terms of delivered packets [10]. SCM, FCM, RWM, and HWM mobility models were used to analyze and evaluate the performance of AODV, OLSR, and GRP protocols over MANETs with varying pause time and speed. The experiment shows that the OLSR protocol provides the best performance regarding throughput, end-to-end delay, and retransmission Attempts [11]. The performance of different ad hoc routing protocols was analyzed and compared based on varying network load, speed, and Mobility. Some of the important performance metrics were calculated. Results indicate that AODV or OLSR should be the first preference when the load of the network is increased [12]. The impact of the network topology and different transport protocols on the performance of MANETs was investigated. Several simulations were done with a varying number of mobile nodes and three various transport protocols [13, 14]. AODV reactive and DSDV proactive routing protocols are analyzed and compared in terms of throughput, packet delivery ratio, routing overhead, and end-to-end delay. Results show that DSDV has a less delay as compare to AODV due to it is proactive nature, while AODV performance is better than DSDV protocol in terms of routing overhead, packet delivery ratio, and throughput [15]. In [16, 17], the authors presented a comparative performance review of various MANET routing protocols including reactive, proactive, and hybrid protocols. The simulated experiments were performed with varying speed of mobile nodes. It is observed that for all routing protocols there are small changes in the values of performance matrices.

This paper aims to interdouce an extensive comparative study of the AODV reactive protocol, DSDV proactive protocol, and AOMDV multi-path routing protocols over MANETs with respect to routing and QoS metrics. Several simulations are done with varying network topology and no of data traffic to analyze the performance of three routings protocols accurately. Six performance metrics have been measured: normalized routing load (NRL), number of routing packet, packet delivery ratio (PDR), and number of packet drop, end-to-end delay, and throughput.

\section{BACKGROUND}

Initially mobile nodes in the ad hoc networks are not aware of network topology they need to discover it. MANET routing protocols control the routing of the packet. These protocols can be classified into a reactive, proactive protocol, hybrid protocols. Figure 1 shows the classification of MANETs routing protocols with examples.

DSDV is one of the most well-known proactive table-driven routing protocols on MANETs [18]. The DSDV routing protocol uses the Bellman-Ford algorithm with certain improvements. Every mobile node maintains a single routing table includes all available destinations along with the number of hops to reach each destination, Sequence numbers used to identify the destination node and the age of the record. DSDV uses both triggered and periodic routing updates to maintain table correspondence. When changes in network topology are detected, Triggered routing updates are used so that all of the routing information is sending as quickly as possible [19].

AODV is one of the most commonly used reactive on-demand MANET routing protocols [20]. This protocol enables self-starting, dynamic, multi-hop routing between mobile nodes and also it is suitable for unicast and multicast routing. AODV allows active mobile nodes to respond to link failures in the topology of the network in a timely manner and allows mobile nodes to take out routes quickly that are actively used for new destinations. Moreover, AODV plays an important role to reduce the number of necessary broadcast messages through the ad hoc network. The AODV uses three different types of the message such as route requests (RREQ), route replies (RREP) and route errors (RERR) to path discovery and maintains the routes [21]. AOMDV is a reactive distance vector routing protocol and an extension for the AODV protocol to computing multiple loops free and link disassemble paths [22]. The routing table for destination nodes includes the 
number of hops to reach the destination along with a list of next hops. In AOMDV, all the next hop neighbors nodes are given the same sequence numbers to keep track of a route. An advertised hop count is maintained by a node for every destination, and this hop count used for sending the destination route advertisements. if a node received any duplicate route advertisement, it defines as an alternative destination path [23, 24].

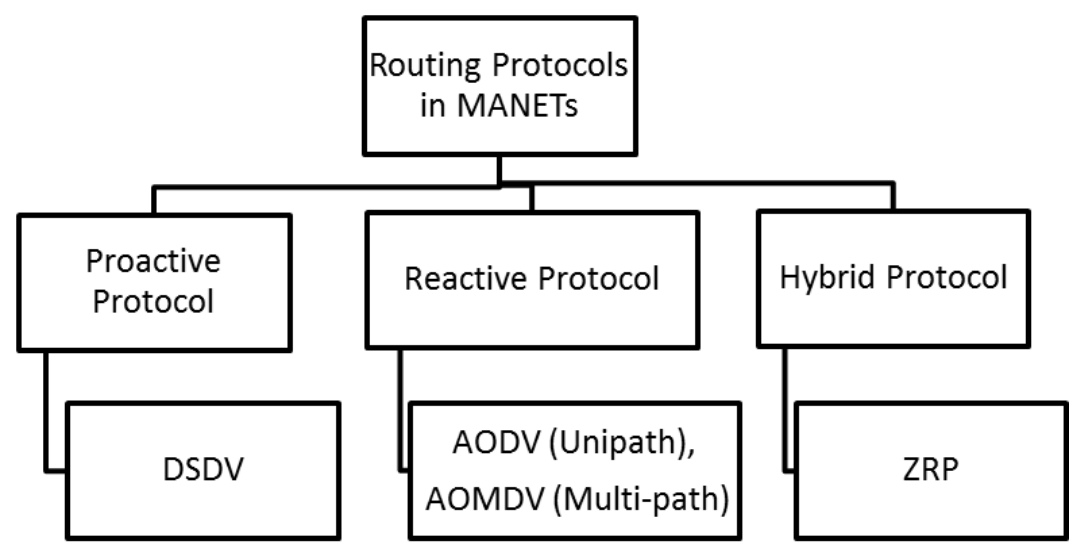

Figure 1. Classification of routing protocol in MANETs

\section{RESEARCH METHOD AND SIMULATION ENVIROMENTS}

\subsection{Simulation enviroments and network topology}

The simulation area dimensions are $800 \times 800 \mathrm{~m}$. The Comparison study includes the simulation of AODV, DSDV, and AOMDV protocols with varying network topology of 10, 20, 30, 40, 50 nodes respectively. The traffic load is set to four connections between source and destination nodes during the whole simulation. mobile nodes are distributed randomly then nodes move with the random way point mobility model to the specific destination and the value of node speed is $50 \mathrm{~m} / \mathrm{s}$. In all simulation cases, the transport protocol is the user datagram protocol (UDP) while the application Traffic type is constant bit rate (CBR). Network simulation NS-3 was used as a simulation tool for all research experiments. The simulation time is $100 \mathrm{sec}$. Figure 2 explains the steps used to execute the simulation experiments.

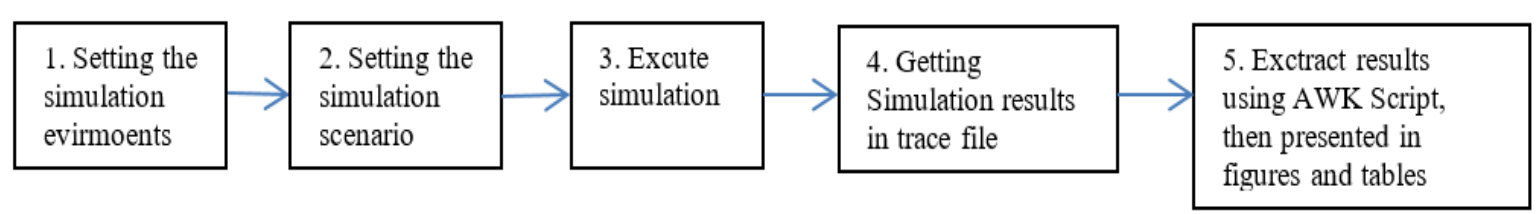

Figure 2. Steps of simulation flow

\subsection{Routing and quality of service $(\mathrm{QoS})$ metrics}

The following routing and QoS metrics are measured in order to analyze and compare the performance of the three routing protocols $[25,26]$.

- Normalized routing load (NRL): it is the ratio between the routing packets and the received packets

- Routing packet: it represents the total number of packet forward by relaying nodes.

- Drop packets: it represents the total number of packet drop in networks due to link failure or congestion in the network.

- Packet delivery ratio: It is the ratio of successfully data packets received by destination to the total number of the data packet sent.

- End-to-end delay: it is the total time a packet needs to be reaching its destination and it measured by (seconds).

- Throughput: it is the total number of received packets in destinations dividing by the duration time and measured by (Kbps). 


\section{SIMULATION RESULTS AND DISCUSSION}

\subsection{Normalized routing load (NRL) and routing packets}

As shown in Figure 3, the NRL of AODV is between 0.15 and 0.4 , whereas that of DSDV is between 0.038 and 0.228 which is slightly lower. At the same time, the NRL of AOMDV significantly affected by the increase in the number of nodes with values between 0.28 and 0.96 . High nodes density with mobility leads to more link failure and frequent route discovery, resulting in congestion control as well as high routing overhead in the network. DSDV achieves the best performance in NRL. Tables 1 and 2 present a comparison of AODV, DSDV, and AOMDV protocols in terms of NRL and routing packets respectively.

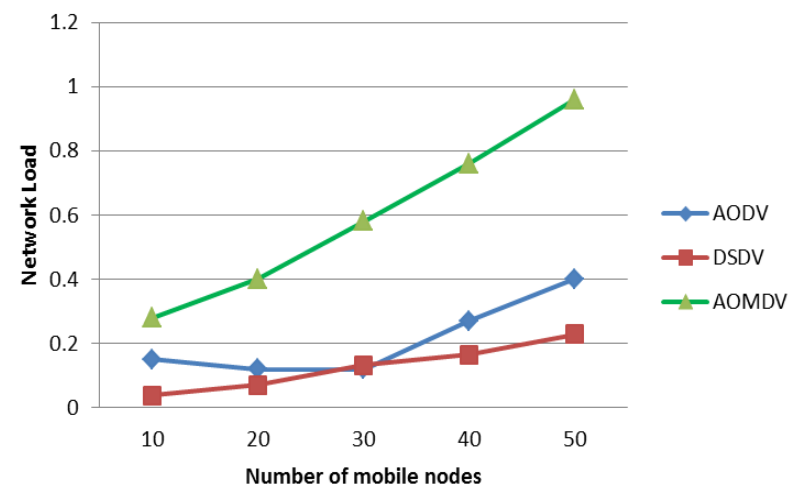

Figure 3. Normalized routing load of AODV, DSDV, and AOMDV

Table 1. Normalized routing load vs number of nodes

\begin{tabular}{cccc}
\hline $\begin{array}{c}\text { Number of } \\
\text { mobile nodes }\end{array}$ & \multicolumn{3}{c}{ Normalized Routing Load (NRL) } \\
AODV & DSDV & AOMDV \\
\hline 10 & 0.15 & 0.038 & 0.28 \\
20 & 0.12 & 0.07 & 0.4 \\
30 & 0.12 & 0.132 & 0.58 \\
40 & 0.27 & 0.164 & 0.76 \\
50 & 0.4 & 0.228 & 0.96 \\
\hline
\end{tabular}

Table 2. Routing packets vs number of nodes

\begin{tabular}{cccc}
\hline Number of & \multicolumn{3}{c}{ Routing Packets } \\
mobile nodes & AODV & DSDV & AOMDV \\
\hline 10 & 509 & 81 & 816 \\
20 & 496 & 178 & 1401 \\
30 & 495 & 371 & 2107 \\
40 & 1110 & 457 & 2725 \\
50 & 1480 & 655 & 3621 \\
\hline
\end{tabular}

\subsection{Drop packets}

The total number of drop packets of AODV, DSDV, and AOMDV is shown in Figure 4 packet drop occurs in ad hoc networks due to the link failure or data rate congestion. The results show that both AODV and AOMDV reactive protocol drops packets less than DSDV proactive protocol because of these protocols route packets on-demand and also if the link fails, both AODV and AOMDV can find a new path to a destination within few seconds. AODV has the fewest number of drop packets as well as it affected slightly by the increase of the mobile nodes. Table 3 shows a comparison of AODV, DSDV, and AOMDV Protocols in terms of drop packets.

\subsection{Packet delivery ratio (PDR)}

Figure 5 shows the packet delivery ratio of AODV, DSDV, and AOMDV protocols under various numbers of mobile nodes. The results show that the packet delivery ratio from AODV is greater than DSDV and AOMDV in 10, 20, 30, and 40 nodes. AODV shows the best performance among the other two routing protocols. In 50 nodes, the packet delivery ratio for AOMDV is very high when compared to the other node density and is equal to $88.03 \%$, while $66.62 \%$ in DSDV, and $86.16 \%$ in AODV. Obviously, the packet delivery ratio of DSDV is very low as compared to AODV and AOMDV on-demand protocols at all nodes densities. The reason behind having a better performance of AODV is that it allows sending packet to stay in the buffer for $30 \mathrm{sec}$ for route discovery, then data packets are sent on that route to the destination. Table 4 displays a comparison of AODV, DSDV, and AOMDV Protocols in terms of PDR.

\subsection{End to end delay}

The end-to-end delay of AODV, DSDV, and AOMDV protocols with varying mobility and pause times are present in Figure 6. It can be noticed that AMODV protocol has the lowest delay when compared to the other two protocols, due to its multipath nature. Furthermore, the AMODV protocol at 20 nodes provides 
the minimum delay that is $16.32 \mathrm{msec}$. On the other hand, DSDV has the highest delay in 30 nodes that is $355.53 \mathrm{msec}$. AODV performance is better than DSDV in high network density 30, 40, 50 nodes with values $232.68 \mathrm{msec}, 131.31 \mathrm{msec}$, and $118.2 \mathrm{msec}$ respectively. The end-to-end delay performance of AODV and AOMDV reactive protocols are affected less than DSDV proactive protocol by an increase the number of nodes. Table 5 presents a comparison of AODV, DSDV, and AOMDV Protocols in terms of end-to-end delay.

\subsection{Throughput}

Figure 7 shows the throughput of the three ad-hoc routing protocols under a varying number of mobile nodes. It can observe that the AODV protocol performs the best compared to the DSDV and AOMDV protocols with a peak throughput of $313.98 \mathrm{kbps}$ at 20 nodes. Contrariwise DSDV has lower performance and suffers a decrease in throughput. This is due to the frequent link changes and connection failures. AOMDV significantly performs better than DSDV which makes it capable of handling high traffic in large networks. Also, it can be observed throughput for AMODV at 50 nodes with value $282.03 \mathrm{kbps}$ is slightly higher than throughput for AODV. Table 6 displays a comparison of AODV, DSDV, and AOMDV Protocols in terms of PDR.

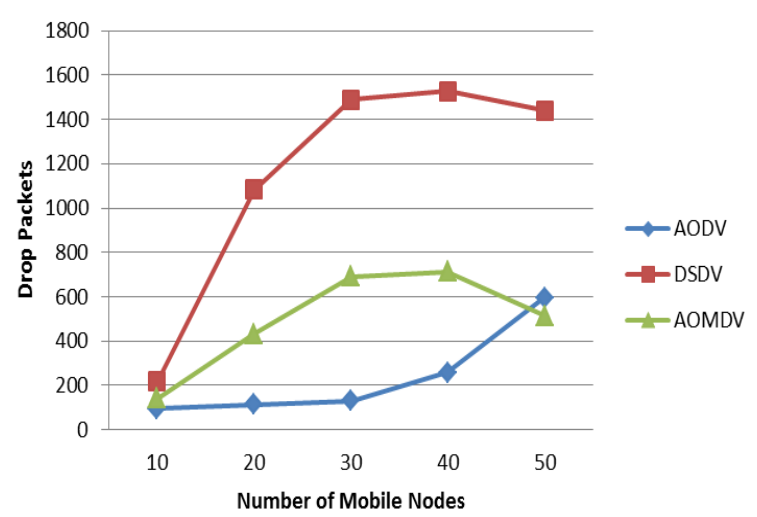

Figure 4. Drop packets of AODV, DSDV, and AOMDV

Table 3. Drop packets vs number of nodes

\begin{tabular}{cccc}
\hline $\begin{array}{c}\text { Number of } \\
\text { mobile nodes }\end{array}$ & \multicolumn{3}{c}{ Drop Packets } \\
& DSDV & AOMDV \\
\hline 10 & 94 & 216 & 140 \\
20 & 113 & 1082 & 432 \\
30 & 129 & 1488 & 690 \\
40 & 258 & 1528 & 713 \\
50 & 595 & 1439 & 515 \\
\hline
\end{tabular}

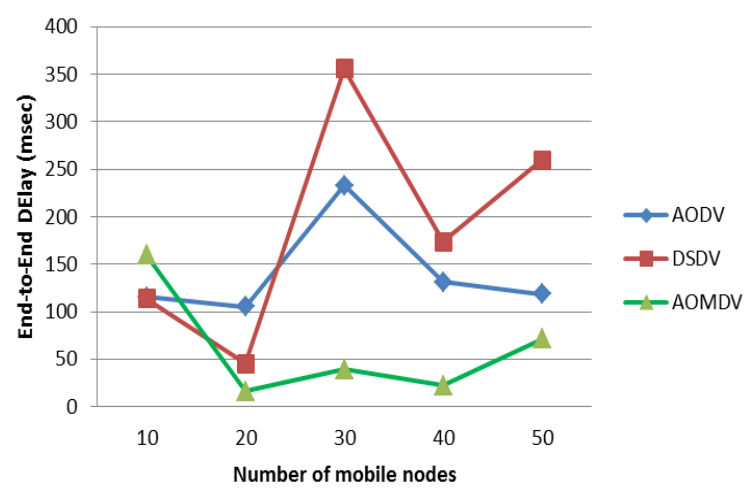

Figure 6. End-to-end delay of AODV, DSDV, and AOMDV

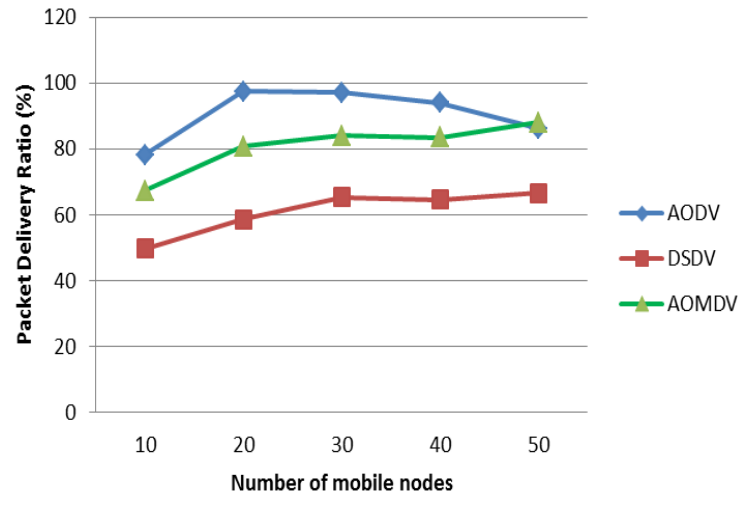

Figure 5. Packet delivery ratio of AODV, DSDV, and AOMDV

Table 4. Packet delivery ratio vs number of nodes

\begin{tabular}{cccc}
\hline $\begin{array}{c}\text { Number of } \\
\text { mobile nodes }\end{array}$ & POcket Delivery Ratio (\%) \\
& 78.15 & 49.78 & 67.4 \\
10 & 97.39 & 58.64 & 80.81 \\
20 & 97.01 & 65.36 & 83.98 \\
30 & 94.03 & 64.54 & 83.44 \\
40 & 86.16 & 66.62 & 88.03 \\
50 & & & \\
\hline
\end{tabular}

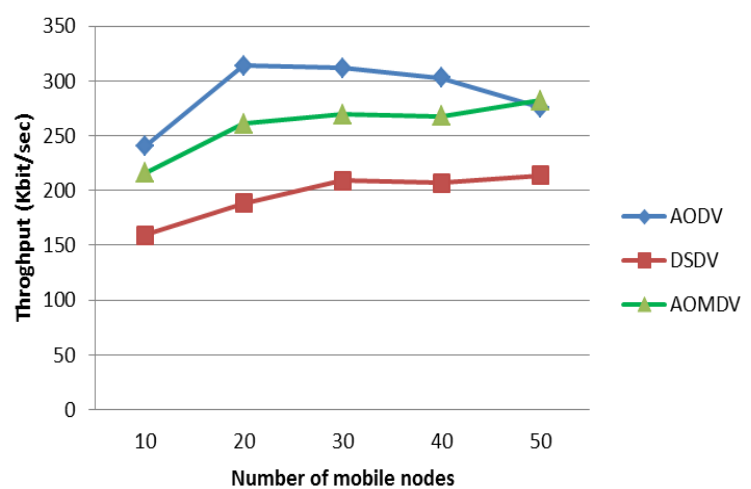

Figure 7. Throughput of AODV, DSDV, and AOMDV 
Table 5. End-to-end delay vs number of nodes

\begin{tabular}{cccc}
\hline Number of & \multicolumn{3}{c}{ End-to-End Delay (msec) } \\
mobile nodes & AODV & DSDV & AOMDV \\
\hline 10 & 115.249 & 113.659 & 159.706 \\
20 & 104.93 & 45.15 & 16.32 \\
30 & 232.68 & 355.53 & 38.97 \\
40 & 131.31 & 173.51 & 22.37 \\
50 & 118.2 & 259.69 & 71.28 \\
\hline
\end{tabular}

Table 6. Throughput vs number of nodes

\begin{tabular}{cccc}
\hline $\begin{array}{c}\text { Number of } \\
\text { mobile nodes }\end{array}$ & \multicolumn{3}{c}{ Throughput (Kbits/sec) } \\
\hline 10 & 240.44 & 159.45 & 215.67 \\
20 & 313.98 & 188.19 & 260.95 \\
30 & 311.52 & 209.04 & 269.29 \\
40 & 302.58 & 207.11 & 267.58 \\
50 & 275.92 & 213.89 & 282.03 \\
\hline
\end{tabular}

\section{CONCLUSION}

MANET is a very important network technology based on a collection of mobile nodes with wireless communication protocols used in several modern applications such as Intelligent Transportation systems, military applications, and flying ad hoc networks. The variety of standard routing protocols in the MANETs make the choice of the most reliable protocol to deliver the information a big challenge. This paper presents an extensive performance study of three different routing protocols AODV, DSDV, and AOMDV in MANETs with varying several network parameters. Results indicate that the end-to-end delay of AOMDV is less than AODV and DSDV. Whiles, AODV performs better than other protocols in terms of normalized routing load, packet delivery ratio, and throughput. In conclusion, network parameters like the number of mobile nodes and the number of connections have a high impact on routing metrics. Future work includes experimental analysis of hybrid protocols and focuses on energy metrics.

\section{REFERENCES}

[1] L. Fratta, M. Gerla, and K. W. Lim, "Emerging trends and applications in ad hoc networks," Annals of Telecommunications, vol. 73, no. 9-10, pp. 547-548, 2018.

[2] T. K. Saini and S. C. Sharma, "Prominent unicast routing protocols for Mobile Ad hoc Networks: Criterion, classification, and key attributes," Ad Hoc Networks, vol. 89, pp. 58-77, 2019.

[3] R. Usha, B. S. Premananda, and K. V. Reddy, "Performance analysis of MANET routing protocols for military applications," 2017 International Conference on Intelligent Computing and Control Systems (ICICCS), Madurai, 2017, pp. 1063-1068.

[4] Y. Jahir, M. Atiquzzaman, H. Refai, A. Paranjothi, and P. G. LoPresti, "Routing protocols and architecture for Disaster Area Network: A survey," Ad Hoc Networks, vol. 82, pp. 1-14, 2019.

[5] S. S. Anjum, R. M. Noor, and M. H. Anisi, "Review on MANET Based Communication for Search and Rescue Operations," Wireless Personal Communications, vol. 94, no. 1, pp. 31-52, 2017

[6] J. Karlsson, L. S. Dooley, and G. Pulkkis, "Secure Routing for MANET Connected Internet of Things Systems," 2018 IEEE 6th International Conference on Future Internet of Things and Cloud (FiCloud), Barcelona, 2018, pp. 114-119.

[7] M. S. Pathan, N. Zhu, J. He, Z. A. Zardari, M. Q. Memon, and M. I. Hussain, "An efficient trust-based scheme for secure and quality of service routing in MANETs," Future Internet, vol. 10, no. 2, pp. 1-16, 2018.

[8] D. N. Kanellopoulos, "QoS Routing for Multimedia Communication over Wireless Mobile Ad Hoc Networks," International Journal of Multimedia Data Engineering \& Management, vol. 8, no. 1, pp. 42-71, 2017.

[9] C. Brill, and T. Nash, "A comparative analysis of MANET routing protocols through simulation," 2017 12th International Conference for Internet Technology and Secured Transactions (ICITST), Cambridge, 2017, pp. 244-247.

[10] M. U. Rahman, and S. Abbas, "Simulation-based analysis of MANET routing protocols using group mobility model," 2016 International Conference on Inventive Computation Technologies (ICICT), Coimbatore, India, 2016, pp. $1-5$.

[11] A. M. Abdullah, E. Ozen, and H. Bayramoglu, "Investigating the impact of mobility models on MANET routing protocols," International Journal of Advanced Computer Science and Applications, vol. 10, no. 2, pp. 25-35, 2019.

[12] N. Sarmah, Y. L. Yang, H. Sharif, and Y. Qian, "Performance analysis of MANET routing protocols by varying mobility, speed and network load," 2015 9th International Conference on Signal Processing and Communication Systems (ICSPCS), Cairns, QLD, Australia, 2015, pp. 1-6.

[13] A. H. Wheeb, "Performance Evaluation of Transport Protocols for Mobile Ad Hoc Networks," Journal of Engineering and Applied Sciences, vol. 13, no. 13, pp. 5181-5185, 2018.

[14] A. H. Wheeb and D. N. Kanellopoulos, "Simulated performance of SCTP and TFRC over MANETs: The impact of traffic load and nodes mobility," International Journal of Business Data Communications and Networking, vol. 16, no. 2 , pp. 69-83, 2020.

[15] A. A. Chavan, D. S. Kurule, and P. U. Dere, "Performance Analysis of AODV and DSDV Routing Protocol in MANET and Modifications in AODV against Black Hole Attack," Procedia Computer Science, vol. 79, pp. 835-844, 2016.

[16] D. S. Sisodia, R. Singhal, and V. Khandal, "A performance review of Intra and inter-group MANET routing protocols under varying speed of nodes," International Journal of Electrical and Computer Engineering (IJECE), vol. 7, no. 5, pp. 2721-2730, 2017.

[17] D. R. Mohsen, and T. Z. Ismaeel, "Estimation and Improvement of Routing Protocol Mobile Ad-Hoc Network Using Fuzzy Neural Network,” Journal of Engineering, vol. 22, no. 7, pp. 142-163, 2016. 
[18] S. Nikam, and B. T., "Delay Analysis of DSDV Protocol using NS 2.34," International Journal of Computer Applications, vol. 134, no. 2, pp. 13-16, 2016.

[19] H. Zemrane, Y. Baddi, and A. Hasbp, "Mobile adhoc networks for intelligent transportation system: Comparative analysis of the routing protocols," Procedia Computer Science, vol. 160, no. 2018, pp. 758-765, 2019.

[20] R. van Glabbeek, P. Höfner, M. Portmann, and W. L. Tan, "Modelling and verifying the AODV routing protocol," Distributed Computing, vol. 29, no. 4, pp. 279-315, 2016.

[21] S. Katiyar, R. Gujral, and B. Mallick, "Comparative performance analysis of MANET routing protocols in military operation using NS2," 2015 International Conference on Green Computing and Internet of Things (ICGCIoT), Greater Noida, India, 2015, pp. 603-609.

[22] I. Baddari, M. A. Riahla, and M. Mezghiche, "A New AOMDV Lifetime Prolonging Routing Algorithm for AdHoc Networks," International Journal of Interdisciplinary Telecommunications and Networking, vol. 11, no. 4, pp. 48-62, 2019.

[23] Ramadoss Prabha and N. Ramaraj, "An improved multipath MANET routing using link estimation and swarm intelligence," EURASIP Journal on Wireless Communications and Networking, vol. 2015, no. 1, pp. 1-9, 2015.

[24] N. Prathviraj, and S. L. Deshpande, "Rough set based QoS enabled multipath source routing in MANET," International Journal of Electrical and Computer Engineering (IJECE), vol. 10, no. 2, pp. 1915-1923, 2020.

[25] A. H. Wheeb, "Performance Analysis of VoIP in Wireless Networks," International Journal of Computer Networks and Wireless Communications (IJCNWC), vol. 7, no. 4, pp. 1-5, 2017.

[26] H. Alani, M. Abdelhaq, and R. Alsaqour, "Dynamic routing discovery scheme for high mobility in mobile adhoc wireless networks," International Journal of Electrical and Computer Engineering (IJECE), vol. 10, no. 4, pp. 3702-3714, 2020.

\section{BIOGRAPHIES OF AUTHORS}

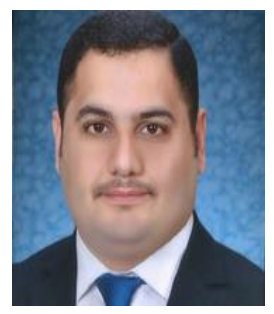

Ali H. Wheeb received a M.Sc. Degree in Computer Engineering from the University of Baghdad, Iraq in 2014. Also, he received an academic promotion (Lecturer) in 2019. Currently, Lecturer Ali is working as a faculty member in the College of Engineering at the University of Baghdad, Iraq. He has published over 10 research articals and one Book. Additionally, he serves as a reviewer for international Conference and high reputation journals published by Springer Nature, Elsevier, IGI, and IAES. His research interests include a computer network, Transport protocols, Routing protocols, MANET, FANETS, wireless network, WSN, IoT, and Network Simulation NS2\& NS3.

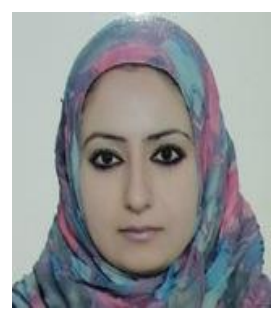

Marwa Taher Naser received a M.Sc. Degree in Computer Engineering from the University of Baghdad, Iraq in 2016. Assestent Lecturer Marwa is working as a faculty member in the College of Engineering at the University of Baghdad, Iraq. She has published 2 research articals. Her research interests include a Routing protocols, MANET, WSN, IoT, and NS2. 